\title{
Mise au point bibliographique, au sujet de la nutrition oligo-minérale des plantes supérieures. Carences et toxicités chez les conifères
}

\author{
E Saur \\ INRA, station d'agronomie, centre de recherches de Bordeaux, \\ BP 131, 33140 Pont-de-la-Maye, France
}

(Reçu le 24 juillet 1989; accepté le 24 octobre 1989)

\begin{abstract}
Résumé - Cette étude bibliographique traite des principaux resultats concernant la nutrition oligo-minérale des plantes supérieures. Le choix est limité aux 4 oligo-éléments dominant en quantité dans les tissus végétaux, c'est-à-dire le cuivre, le manganèse, le zinc et le bore. Chaque monographie concerne la présence de l'élément dans la roche-mère, les différentes formes présentes dans les sols et les phénomenes d'absorption racinaire et de transfert dans la plante. Un rapide résumé des fonctions biochimiques de chaque élément permet de préciser son importance dans la physiologie des végétaux. Dans une deuxième partie sont exposés les cas de carence en oligo-éléments et de toxicité de ceux-ci décrits pour des peuplements forestiers de conifères.
\end{abstract}

nutrition des plantes / cuivre / manganèse / zinc / bore / conifère

Summary - Trace element nutrition of plants. Deficlency and toxiclty in conifers. In this review on the trace-element nutrition of plants, only copper, manganese, zinc and boron are considered. For each element, we discuss: sources and availability to plants, uptake and distribution within the plant. A summary of the biochemical roles of trace elements in plant metabolism is given in order to explain their consequences on growth and development. In the second part we discuss trace-element deficiency and toxicity in conifers.

plant nutritlon / copper / manganese / zinc / boron / conlfers

\section{INTRODUCTION}

Les études sur les oligo-éléments dans les espèces végétales et, plus particulièrement, les plantes cultivées, ont été très nombreuses depuis le début de ce siècle. Pour des raisons propres à la physiologie et à la longévité des ligneux, les essences forestières n'ont fait l'objet que de peu de travaux, souvent suscités par des cas de carence graves. 
Nous nous proposons, dans une première partie, de faire une mise au point sur chacun des 4 oligo-éléments dominant en quantité dans les tissus végétaux $(\mathrm{Cu}, \mathrm{Mn}, \mathrm{Zn}, \mathrm{B})$ avec leur comportement dans les sols et les plantes. Dans la seconde partie, nous avons tenté une synthèse aussi exhaustive que possible des travaux consacrés aux problèmes sylvicoles liés à des carences en oligo-éléments ou des toxicités de ces éléments.

\section{TRANSFERT DES OLIGO-ÉLÉMENTS DU SOL À LA PLANTE ET FONCTIONS PHYSIOLOGIQUES}

\section{Le cuivre}

\section{État du cuivre dans le sol}

La quantité de cuivre du sol disponible pour les végétaux dépend des réserves présentes dans la roche-mère, et surtout des conditions physico-chimiques régnant dans le sol.

\section{Le cuivre dans la roche-mère}

La concentration moyenne en cuivre de la croûte terrestre est d'environ $70 \mathrm{mg} / \mathrm{kg}$ (Hodgson, 1963), celle des roches du territoire national français $20 \mathrm{mg} / \mathrm{kg}$ (Aubert et Pinta, 1971). On trouve le cuivre surtout sous forme de sulfures simples ou complexes (Sauchelli, 1969; Krauskopf, 1972).

La teneur de la roche-mère dépend de son origine géologique (Aubert et Pinta, 1971) :

- roche éruptive basique : $100-200 \mathrm{mg} / \mathrm{kg}$;

- roche éruptive acide : 10-20 mg/kg;

- roche métamorphique, argile, loess : 30-40 $\mathrm{mg} / \mathrm{kg}$;

- grès, sable, calcaire : 3-10 mg/kg.
Des résultats agronomiques ont montré que les carences en cuivre apparaissaient sur des roches-mères pauvres en cet élément, comme l'attestent les exemples observés sur les sols granitiques de Bretagne (Coppenet et Jolivet, 1952), les sols sableux des Landes de Gascogne (Redlich, 1954) et les sols gréseux de Basse-Normandie et du Bas-Maine (Duval, 1963). Le seuil de carence pour un sol serait de 7 à $8 \mathrm{mg} / \mathrm{kg}$ de Cu total (Duval et Maurice, 1970).

Cette constatation générale, qui met en parallèle la pauvreté en cuivre d'une roche-mère et la déficience des végétaux en cet élément, est insuffisante pour expliquer dans le détail la nutrition des plantes. En effet, la teneur totale d'un sol en un élément refiète mal sa biodisponibilité ${ }^{\star}$ (Juste, 1988). Mac Laren et Crawford (1973) ont montré que, dans les sols du Royaume Uni, une forte proportion du cuivre total était indisponible pour les végétaux.

Pour tenter de définir la biodisponibilité d'un élément, il est donc nécessaire de distinguer les différents états de cet élément dans un sol.

\section{Les différentes formes du cuivre dans le sol}

Le cuivre, comme tout élément du sol, peut se trouver dans l'un des 4 compartiments suivants : minéraux primaires ou secondaires, composés organiques, formes échangeables sur les colloïdes du sol et solutions du sol (Guillet, 1980; fig 1).

La forme $\mathrm{Cu}^{2+}$ est dominante dans les sols, en effet $\mathrm{Cu}^{+}$est instable aux températures ordinaires et aux concentrations supérieures à $10^{-7} \mathrm{~mol} \cdot \mathrm{f}^{-1}$ (Loué, 1986).

La majeure partie du cuivre est généralement incluse dans les mailles

\footnotetext{
* Aptitude d'un élément à être transféré d'un compartiment quelconque du sol vers un organisme vivant
} dans ce dernier. 
cristallines des minéraux primaires ou secondaires (Mengel et Kirkby, 1982). Le reste du cuivre, potentiellement disponible pour les plantes, est fortement adsorbé sur les surfaces d'echange du sol (argiles: Muller, 1960; hydroxydes de fer : Mac Bride et Blaziak, 1979) ou complexé avec la matière organique (acides humiques et fulviques : Hodgson et al, 1966; Delas, 1967; Schnitzer et Skinner, 1966).

La concentration du cuivre dans la solution du sol est le résultat d'un équilibre chimique entre les différentes phases qui sont liées par des réactions acido-basiques, d'oxydo-réduction, de précipitation, de dissolution, d'absorption et de complexation (Lepp, 1979).

En milieu abiotique, la mobilité et l'assimilabilité du cuivre sont conditionnees par les propriétés physico-chimiques du sol; l'élévation du $\mathrm{pH}$ diminue fortement l'assimilabilité du cuivre (Drouineau et Mazoyer, 1962).

L'intervention de la microflore joue un rôle important dans l'immobilisation du cuivre dans les couches superficielles de certains sols (Kabata-Pendias et Pendias, 1984).

Le mouvement des ions dans le compartiment liquide du sol est conditionné par 2 processus principaux : la diffusion et le flux de masse (convection). Le transfert du cuivre vers la racine semblerait se réaliser principalement par le flux de masse (Hodgson et al, 1966).

\section{Absorption du cuivre par les racines et rôle dans la physiologie du végétal}

\section{Absorption du cuivre}

Le cuivre est prélevé en faible quantité par la plante, la teneur en cet élément de la majorité des végétaux étant

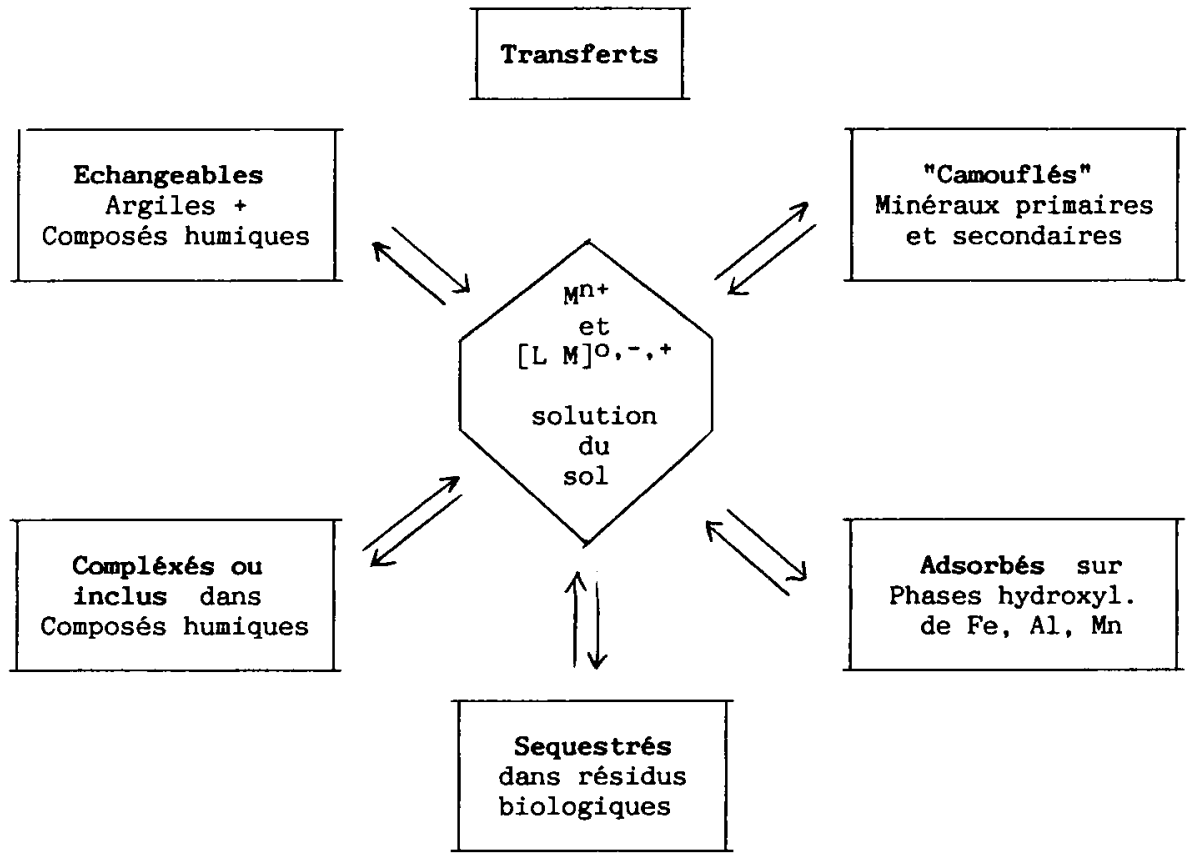

Fig 1. État des métaux dans les sols (d'après $B$ Guillet et al, 1980); $M=$ métal; $L=$ ligand 
comprise entre 2 et $20 \mathrm{mg} / \mathrm{kg}$ de poids sec. Dans les tissus racinaires, le cuivre est presque complètement sous forme complexée mais il est probable qu'il pénètre à l'état élémentaire dans les cellules de la racine (Kabata-Pendias et Pendias, 1984).

Le prélèvement du cuivre par la plante est un processus actif, affecté par des inhibiteurs métaboliques (Dokiya et al, 1964).

La cinétique d'absorption du cuivre à des doses croissantes est du type Michaelis-Menten, elle a été décrite par Bowen (1969) sur feuilles de canne à sucre, Nielsen (1976) sur l'orge et Cathala et Salsac (1975) sur maîs et tournesol.

Des phénomènes de prélèvement passif pourraient intervenir pour des doses importantes de cuivre en solution (Kabata-Pendias et Pendias, 1984); Cathala et Salsac (1975) ont suggéré le même mécanisme dans le cas de racines excisées.

Mais l'absorption du cuivre et des autres métaux lourds est encore très discutée et le caractère michaëlien de la cinétique pourrait traduire la succession de différents mécanismes qui n'impliquent pas forcément que l'absorption soit réalisée par des transporteurs métaboliques (Harrison et al, 1979).

L'absorption du cuivre est principalement liée à sa concentration sous forme assimilable dans le sol (Mengel et Kirkby, 1982), mais aussi à l'inhibition compétitive du zinc qui utilise les mêmes sites d'absorption racinaire (Schmid et al, 1965; Bowen, 1969).

Keller et Deuel (1958) ont montré que le cuivre est capable de chasser de nombreux autres ions des sites d'échange racinaire. L'intensité de la rétention du cuivre sur les parois cellulaires et dans les espaces intercellulaires est liée à la capacité d'é- change racinaire (Cathala et Salsac, 1975). Ceci explique l'accumulation de cuivre dans les racines des végétaux observée par Russ (1958), Hill (1973) et Jarvis (1978).

\section{Migration du cuivre dans la plante}

Les mouvements du cuivre entre les différents tissus de la plante sont déterminants pour son utilisation. Ils prennent une importance particulière dans l'alimentation des plantes pérennes caractérisées par des mécanismes physiologiques propres: les vagues de croissance des rameaux et des racines, la mise en réserve des éléments dans les tissus et leur redistribution dans la plante.

Les mouvements du cuivre dans la plante sont généralement assez faibles. La mobilité dépend principalement des teneurs dans la plante (Loneragan, 1975), mais aussi de l'intensité du métabolisme azoté. En effet, Tiffin (1972) et Loneragan (1975) ont montré que le cuivre est excrété par les cellules racinaires dans les vaisseaux du xylème et du phloème sous forme anionique complexée avec des composés azotés (acides aminés). L'excrétion de ces exsudats mobiles dans les vaisseaux serait le processus clé de la nutrition en cuivre des plantes.

La baisse des concentrations dans les feuilles âgées est parallèle à leur sénescence et à leur appauvrissement en azote. Elle semble dépendre de l'hydrolyse des protéines auxquelles le cuivre est lié (Loneragan et al, 1980).

\section{Fonctions biochimiques du cuivre}

Les fonctions biochimiques du cuivre dans la physiologie des végétaux ont fait l'objet de nombreuses études et peuvent se résumer ainsi :

- le cuivre est principalement complexé avec des composés organiques 
de poids moléculaire faible et avec des protéines;

- le cuivre intervient dans la composition de certaines enzymes;

- le cuivre joue un rôle fondamental dans certains processus physiologiques tels que la photosynthèse, la respiration, le transfert des sucres, la réduction et la fixation de l'azote, le métabolisme protéique et le métabolisme des parois cellulaires;

- le cuivre conditionne la perméabilité à l'eau des vaisseaux du xylème et donc, le contrôle des transferts hydriques dans la plante;

- le cuivre régule l'anabolisme de I'ADN et de I'ARN, une carence en cuivre affecte considerablement la reproduction des plantes;

- le cuivre intervient dans le mécanisme de resistance aux maladies.

La plupart des processus affectés par la déficience en cuivre résultent d'un effet indirect de celui-ci (KabataPendias et Pendias, 1984). Cependant, il exerce un rôle direct sur le processus de lignification (Rahimi et Bussler, 1974) au travers d'enzymes comme la laccase et les péroxydases.

Sur les espèces forestières, les carences en cuivre provoquent des déformations des axes, liées à une diminution de la lignification du bois, comme l'ont montré Downes et Turvey (1986) sur Pinus radiata.

\section{Interaction phosphore-cuivre}

Des études sur l'interaction entre les nutritions en phosphore et en cuivre ont été réalisées, soit à la suite de l'utilisation prolongée de fortes doses d'engrais phosphaté, soit lors d'expériences conduites en milieu nutritif artificiel (tableau I).

Bingham et al (1958) ont les premiers mis en évidence sur citronnier des carences graves en cuivre, in-

Tableau I. Revue bibliographique concernant les carences en cuivre induites par le phosphore.

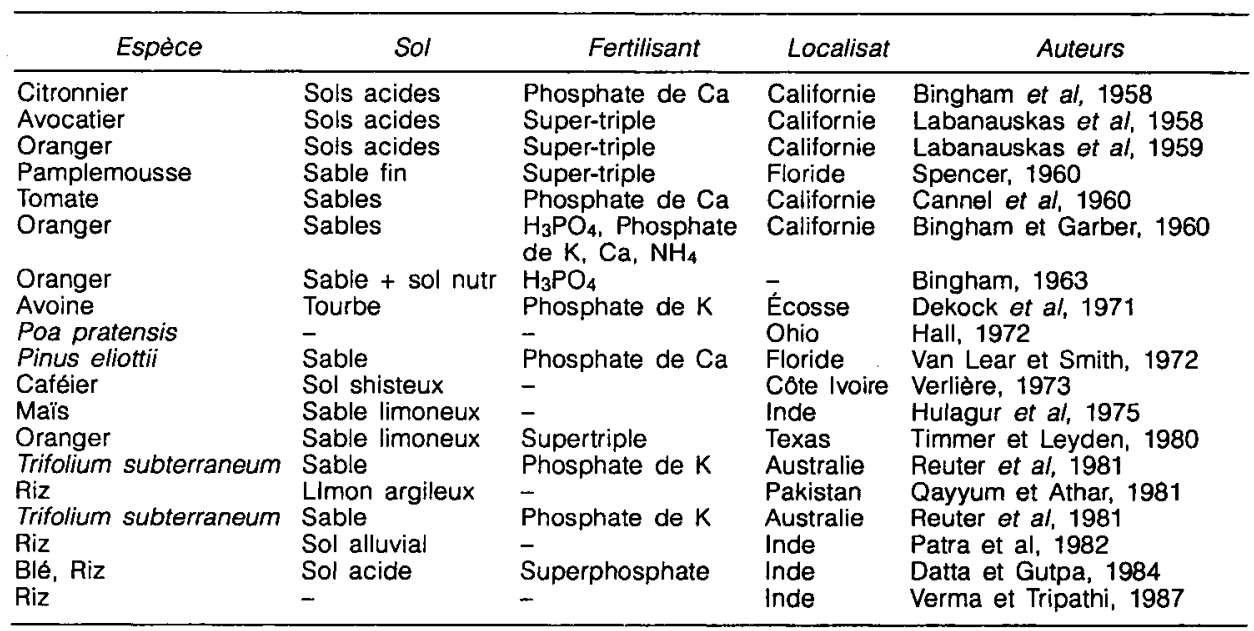


duites par l'apport d'acide phosphorique à 9 sols de Californie : les amendements cupriques suppriment les symptômes de carence et améliorent la croissance. Bingham et Garber (1960) ont observé une baisse significative des concentrations en $\mathrm{Cu}$ dans les semis d'orangers soumis à des fertilisations à base d'acide phosphorique et de phosphates de potassium, d'ammonium et de calcium, et cela malgré une augmentation des teneurs en cuivre soluble à l'eau dans les 3 sols expérimentés. Cette interaction est confirmée par Bingham (1963) pour des semis d'oranger en solution nutritive sur sable.

En résumé, la carence en cuivre induite par le phosphate a été décrite sur pin, citronnier, oranger, pamplemousse, avocatier, caféier, tomate, trèfle, poa, blé, maïs et riz (tableau 1). Elle apparaît toujours sur des sols très pauvres en cuivre et le plus souvent acides.

A l'inverse, l'action inhibitrice des fortes doses de cuivre sur le prélèvement du phosphore par la plante a été démontrée par Greenwood et Hallsworth (1960) sur Trifolium subterraneum, Spencer (1966) sur le citronnier et Patra et al (1982) sur le riz. Elle serait la conséquence de l'effet toxique du cuivre sur le métabolisme racinaire ou mycorhizien (Graham et al, 1986, sur citronnier).

L'effet favorable des mycorhyzes à vésicules et arbuscules sur la nutrition des plantes en cuivre a été démontré à de nombreuses reprises par Lambert et al (1979) sur soja et maïs, Timmer et Leyden (1980) sur citronnier, Gildon, Gildon et Tinker (1983) sur poireau et maïs, Krishna et Bagyaraj (1984) sur arachide, Hall et al (1984) sur raygrass, Pacovsky et al (1986a et b) sur soja et sorgho, Colozzi-Filho et Siqueira
(1986) sur caféier, Sasa et al (1987) sur poireau et Kucey (1987) sur fève.

Timmer et Leyden (1980) ont suggéré que la réduction des teneurs en cuivre dans les plantes en présence de fortes doses de phosphore était liée à la réduction de l'exploitation du sol par les mycorhizes et, en conséquence, à celle de l'absorption du métal. Le phosphore exerce un effet dépressif sur l'inoculation des racines par les mycorhizes et il entrerait en compétition avec le cuivre pour les sites d'absorption (Gildon et Tinker, 1983).

Lambert et al (1979) ont démontré en effet que l'interaction $\mathrm{P}-\mathrm{Cu}$ ne s'exprimait pas en l'absence de mycorhizes à vésicules et arbuscules sur soja et poireau.

Mais, à notre connaissance, il n'existe pas de travaux démontrant l'action des ectomycorhizes des arbres forestiers sur l'absorption du cuivre, à l'exception d'une expérience de Sidle et Shaw (1987) qui signalent une baisse des teneurs en cuivre des aiguilles de Picea sitchensis après inoculation par Laccaria laccata.

\section{Le manganese}

\section{État du manganèse dans le sol}

\section{Le manganèse dans la roche-mère}

Le manganèse est l'élément-trace le plus abondant de la lithosphère. La teneur moyenne en manganèse de l'écorce terrestre est de l'ordre de 900 à $1000 \mathrm{mg} / \mathrm{kg}$ (Kovda et al, 1964), les teneurs courantes dans les sols variant de 200 à 3000 mg/kg (Swaine, 1955). Aubert et Pinta (1971) ont noté des teneurs (en $\mathrm{mg} / \mathrm{kg}$ ) : élevées dans les roches éruptives basiques (10002 000), moyennes dans les calcaires (400-600) et faibles dans les sables 
(20-500); elles sont très variables dans les roches éruptives acides et les roches métamorphiques (200-1200). On trouve le manganèse en abondance dans les séries géologiques ferro-magnésiennes.

Les minéraux manganiques sont surtout des oxydes (pyrolosite, manganite, haussmanite) mais aussi des carbonates et des silicates.

\section{Les différentes formes de manganèse dans le sol}

Le manganèse du sol se présente sous plusieurs formes: inclus dans un minéral, complexé par la matiere organique, echangeable et en solution, le manganèse soluble pouvant être, soit sous forme ionique $\left(\mathrm{Mn}^{2+}\right)$, soit combiné avec des molécules organiques solubles. L'équilibre entre ces formes est dominé par les réactions d'oxydation à la surface des particules minerales du sol et les réactions d'adsorption sur les minéraux argileux ou la matière organique. Le $\mathrm{pH}$ et les conditions oxydoréductrices du sol sont les 2 facteurs principaux régulant cet équilibre. En conséquence, l'assimilabilité du manganèse est fortement améliorée par la baisse des $\mathrm{pH}$, l'ion $\mathrm{Mn}^{2+}$ prélevé par les végétaux étant fortement dominant aux $\mathrm{pH}$ acides.

La richesse du sol en matière organique diminue la biodisponibilité de cet élément. D'autre part, une forte hydromorphie du sol, qui entraîne des conditions réductrices drastiques est susceptible d'augmenter fortement le taux de manganese assimilable, provoquant des risques de toxicité pour le végétal (Meek et al, 1968; Lal et Taylor, 1970).

En marge de ces phénomènes purement physico-chimiques, il faut citer l'activité microbiologique des sols, reconnue pour être en grande partie res- ponsable de l'oxydation, ou de la réduction des composés manganiques. Leur activité est dépendante du $\mathrm{pH}$, les micro-organismes sont oxydants dans les sols neutres ou basiques (Meek et al, 1968), mais ils peuvent aussi s'avérer réducteurs en milieu anoxique (Lucas et Knezek, 1972).

\section{Absorption du manganèse par les ra- cines et rôle de celui-ci dans la phy- siologie du végétal}

\section{Absorption du manganèse}

D'après Halstead et al (1968), le manganèse est absorbé, soit sous forme de complexe soluble, soit après dissociation, sous forme $\mathrm{Mn}^{2+}$. Godo et Reisenauer (1980) ont montré que les exsudats racinaires de blé, dans des sols de $\mathrm{pH} 5,5-6,0$, avaient un effet sur la réduction en $\mathrm{Mn}^{2+}$ du manganèse du sol et sur sa complexation, le rendant ainsi plus disponible pour la racine.

Le manganèse est prélevé de manière active par des transporteurs membranaires sensibles aux poisons métaboliques (Maas et al 1968; Moore, 1972; Halstead et al, 1968). II semble que les transporteurs soient les mêmes que ceux du magnésium et du calcium. Lohnis (1960) et Maas et al (1969) ont mis en évidence une compétition entre $\mathrm{Mn}^{2+}$ et $\mathrm{Mg}^{2+}$. En tout état de cause, le manganèse est transporté assez rapidement vers le xylème car il réagit peu avec les ligands organiques insolubles dans le tissu racinaire (KabataPendias et Pendias, 1984).

Migration du manganèse dans la plante Le manganèse est transporté assez rapidement dans le xylème, vers les parties en croissance (Amberger, 1973). II semble que la forme cationique libre soit dominante dans ce transport (Bremmer et Knight, 1970; Tiffin, 1977; 
Tinker, 1981). Par contre, la redistribution par le phloème est peu intense (Van Goor, 1974). Les quantités transportées dans la sève du phloème sont faibles et une partie du manganèse serait liée à des petites molécules organiques (Van Goor et Wiersma, 1976).

\section{Fonctions biochimiques du manganèse}

Parmi les métaux de transition (manganèse, fer, cuivre, zinc), le manganèse présente les plus faibles constantes de stabilité lors de la formation de complexes (Clarkson et Hanson, 1980). II peut remplacer le magnésium dans de nombreuses réactions enzymatiques et il intervient généralement comme constituant dans des métallo-protéines, comme site actif de liaison ou dans les systèmes d'oxydo-réduction.

On retrouve le manganèse dans les étapes importantes du métabolisme des protéines et des glucides, son rôle dans le métabolisme lipidique n'étant pas clairement établi. II intervient dans la réduction des nitrates au niveau de l'hydroxylamine réductase. II a également un rôle déterminant dans le transport photosynthétique d'électrons au niveau de l'oxygène accepteur final; en cas de carence manganique, c'est le système photosynthétique qui est altéré le premier.

\section{Le Zinc}

\section{État du zinc dans le sol}

\section{Le zinc dans la roche-mère}

La teneur moyenne en zinc de la lithosphère est en général de $50 \mathrm{mg} / \mathrm{kg}$ environ (Kovda et al, 1964) et les variations ne sont pas très importantes: de 10 a $150 \mathrm{mg} / \mathrm{kg}$.

La teneur en zinc de la roche-mère dépend de son origine géologique (Aubert et Pinta, 1971) :
- roches éruptives basiques $70-130 \mathrm{mg} / \mathrm{kg}$; - roches métamorphiques et argiles sédimentaires, $80 \mathrm{mg} / \mathrm{kg}$ en moyenne; - roches éruptives acides, 50-60 mg/kg; - limons loessiques, argiles glaciaires, $30-40 \mathrm{mg} / \mathrm{kg}$;

- roches carbonatées, grès, $15-20 \mathrm{mg} / \mathrm{kg}$.

Dans les roches, le zinc se trouve principalement sous forme de sulfures (sphalérite), carbonates (smithsonite) et de silicates (willemite). On le rencontre comme ion de remplacement dans les mailles cristallines des roches ferro-magnésiennes (augite, horblende, biotite). II est adsorbé entre les feuillets des argiles minérales ou en substitution du magnésium (montmorillonite).

\section{Les différentes formes $d u$ zinc dans le sol}

L'alteration de la roche-mère produit des ions $\mathrm{Zn}^{2+}$ mobiles, particulierement en milieu acide et oxydant. Le zinc est ensuite fortement adsorbé sur les argiles et la matière organique. Dans ces conditions, la solubilité du zinc est nettement abaissée avec l'élévation du pH (Dolar et Keeney, 1971). Zyrin et al (1976) ont montré que le zinc coprécipitait avec les hydroxydes de fer et d'aluminium.

D'après Kabata-Pendias et Pendias (1984), les minéraux argileux, les hydroxydes et le $\mathrm{pH}$ sont les facteurs principaux intervenant dans la solubilité du zinc. La complexation avec la matière organique et la cristallisation en hydroxydes, carbonates ou sulfides présente une importance moindre.

Le déplacement du zinc vers la surface racinaire dépend peu des mouvements d'eau, il se fait surtout par diffusion engendrée par les très faibles teneurs en zinc du liquide interstitiel au voisinage immédiat de la racine (Wilkinson et al, 1968; Elgawhary et al, 
1970). La présence de mycorhizes augmente notablement ce phénomène.

\section{Absorption du zinc par les racines et rôle dans la physiologie du végétal}

\section{Absorption du zinc}

L'absorption du zinc est en grande partie sous contrôle métabolique. De nombreux auteurs ont montré l'effet d'inhibiteurs sur le prélèvement (Schmid et al, 1965; Chaudhry et Loneragan, 1972; Giordano et al, 1974). Le phosphore, le fer et le cuivre peuvent entrer en compétition avec le zinc dans les processus d'absorption et de transfert.

\section{Migration du zinc dans la plante}

Les teneurs en zinc de la sève du $x y$ lème sont généralement supérieures à celles du liquide prélevé par les racines. Le zinc semble être transporté dans cette sève sous forme ionique (Tiffin, 1967) ou lié à des acides organiques (White et al, 1981). Par contre, dans la sève du phloème, il est véhiculé sous forme de complexe anionique (Van Goor et Wiersma, 1976).

Le zinc est un élément peu mobile et, en cas de consommation "de luxe», il tend à s'accumuler dans les racines (Loneragan, 1977). Massey et Loeffel (1967) ont montré, sur maïs, la possibilité d'une remobilisation du zinc foliaire au moment de la formation de l'épi, mais cette remobilisation ne se manifeste plus si la plante est carencée (Riceman et Jones, 1958).

\section{Fonctions biochimiques du zinc}

Dans le végétal, le zinc n'intervient pas dans les réactions d'oxydo-réduction, ce sont surtout ses propriétés de cation bivalent et sa forte propension a former des complexes tétraédriques qui pré- valent (Clarkson et Hanson, 1980). Le zinc agit soit comme constituant, soit comme cofacteur d'enzyme.

Le zinc est un constituant de l'alcool déhydrogénase, de la super-oxyde dismutase, de l'anhydrase carbonique et de l'ARN polymérase (Vaughan et al, 1982; Sandmann et Boger, 1983). Par activation d'autres enzymes (fructose1,6-biphosphatase, PEP carboxylase), il intervient dans le métabolisme glucidique et dans le métabolisme protéique. Son rôle est généralement connu dans la synthèse du tryptophane et de l'acide indole-acétique. La baisse des teneurs en AIA dans une plante carencée en zinc explique les symptômes d'arrêt de croissance et de faible élongation des feuilles.

\section{Le Bore}

\section{État du bore dans le sol}

\section{Le bore dans la roche-mère}

La teneur moyenne en bore de l'écorce terrestre est de $50 \mathrm{mg} / \mathrm{kg}$ environ (Kovda et al, 1964). Cette teneur dépend fortement de la proportion des roches sedimentaires d'origine marine, qui contiennent jusqu'à $500 \mathrm{mg}$ de bore $/ \mathrm{kg}$. Les roches continentales sont beaucoup plus pauvres (Aubert et Pinta, 1971):

- roches métamorphiques, roches sédimentaires continentales, 5-12 mg/kg; - roches éruptives acides, 3-10 mg/kg; - roches éruptives basiques, $1-5 \mathrm{mg} / \mathrm{kg}$.

Le minéral le plus riche en bore est la tourmaline, où le bore intervient, ainsi que dans d'autres silicates, comme ion de remplacement du silicium dans les structures cristallines tétraédriques. 


\section{Les différentes formes du bore dans le sol}

Au cours de l'altération des roches, le bore passe facilement en solution sous forme d'anions $\left(\mathrm{BO}_{2}^{-}, \mathrm{B}(\mathrm{OH})_{4}{ }^{-}\right)$.

Il est abondamment absorbé par les argiles et les substances organiques. La forme la plus courante dans la solution du sol est l'acide borique ou l'ion borate $\mathrm{B}(\mathrm{OH})_{4}{ }^{-}$.

L'absorption du bore par les hydroxydes de fer et d'aluminium est un mécanisme important qui commande en grande partie la solubilité de cet élément dans le sol (Sims et Bingham, 1968). La rétention du bore par la matière organique est prépondérante en milieu acide où les colloïdes humiques formeraient la réserve principale en bore, les micro-organismes interviennent en le libérant progressivement (Parker et Gardner, 1982).

\section{Absorption du bore par les racines et rôle dans la physiologie du végétal}

\section{Absorption du bore}

Les formes solubles du bore sont facilement assimilables par les plantes et l'acide borique non dissocié serait la forme d'absorption privilégiée. La propension de l'acide borique à former des complexes avec les polysaccharides des parois végétales joue un rôle important dans l'absorption passive (Tanaka, 1967; Thellier et al, 1979). Les concentrations en bore dans les racines dépassent légèrement celles du liquide nutritif et l'absorption se fait principalement par flux de masse (Oertli, 1963; Bingham et al, 1970; Bowen et Nissen, 1976). Des processus d'absorption métabolique ont été mis en évidence (Moore, 1972) mais ils sont mineurs et s'expriment pour de faibles concentrations externes (Nissen, 1974).

\section{Migration du bore dans la plante}

Le bore est transporté dans la sève du xylème, sous forme inorganique, ou lié a des sucres. Son transport est très etroitement dépendant du flux de transpiration (Michael et al, 1969) et est souvent de faible intensité. Contrairement aux autres oligo-éléments, le bore est faiblement absorbé par les tissus limitrophes du xylème pendant son ascension dans la plante (Van Goor, 1974). Le bore a donc tendance à s'accumuler dans les parties supérieures du végétal à forte activité transpiratoire (Wilkinson, 1957; Jones, 1970).

La redistribution par le système phloémique, en particulier vers les zones de croissance, est très limitée (Raven, 1980). Ceci explique l'apparition des premiers symptômes de carence au niveau des zones méristématiques et la très faible autonomie du végétal mis en situation de carence.

\section{Fonctions biochimiques du bore}

Contrairement aux 3 autres oligo-éléments passés en revue, le bore n'intervient pas comme constituant des enzymes du métabolisme végétal. Le rôle du bore dans les mécanismes biochimiques n'est pas bien connu, mais de nombreuses études ont porté sur les conséquences de sa carence. II en ressort que le bore est impliqué dans 4 processus physiologiques importants :

- le métabolisme glucidique et le transport transmembranaire des sucres, comme en témoigne le caractère aigu et spectaculaire des carences observées dans les organes fortement accumulateurs de sucre (racines de betterave, tubercules de pomme de terre);

- la formation des parois cellulaires (pectines);

- l'activité méristématique;

- la synthèse des acides nucléiques et de phytohormones (Kabata-Pendias et Pendias, 1984). 


\section{CARENCE ET TOXICITÉ EN OLIGO- ÉLÉMENTS CHEZ LES CONIFÈRES}

\section{Le cuivre}

Le cuivre est un oligo-élément dont le caractère indispensable à la vie des plantes est admis par tous depuis fort longtemps (Mc Hargue, 1925; Sommer, 1931; Lipman et Mac Kinney, 1931), mais les études décrivant son rôle spécifique chez les arbres forestiers sont peu nombreuses. Néanmoins, des travaux anciens ont permis de caracteriser des carences ou des toxicités en cuivre provoquant des troubles physiologiques chez les arbres concernés.

Benzian et Warren (1956) ont découvert des carences en cuivre, dans une pépinière sur sol sableux et acide, exprimées par des brûlures de l'extrémité des aiguilles sur l'épicéa de Sitka. Ces carences ont pu être corrigées par des apports d'engrais cuivrés.

En 1961, Hall a constaté des carences sur Pinus radiata et $P$ pinaster en Australie. Également, Materna (1962) a défini des seuils de carences pour des teneurs foliaires de $2 \mathrm{mg} / \mathrm{kg}$ pour l'épicéa commun et de 2,3 $\mathrm{mg} / \mathrm{kg}$ pour l'épicéa de Sitka.

L'exemple le plus célèbre de carence en cuivre sur conifère est celui décrit par Van Goor (1963 et 1965 a et b) en Hollande sur le douglas, qui adopte un aspect pleureur avec un manque de rigidité de tous les axes feuillés lorsque les teneurs foliaires en cuivre atteignent $2 \mathrm{mg} / \mathrm{kg}$. Une fumure déséquilibrée en azote et phosphore a été mise en cause, ainsi qu'une minéralisation rapide de la matiere organique à la suite du labour de ces sols a humus brut (carence en $\mathrm{Cu}$ induite par N-P). De son côté, Penningsfeld (1964) a constaté le même genre de symptômes sur des jeunes plants de mélèze.

Ahrens (1965) a mesuré des teneurs normales en cuivre qui oscillent entre 3 et $12 \mathrm{mg} / \mathrm{kg}$ dans les aiguilles de résineux, mais il n'a pu établir de relation entre la classe de fertilité des stations et la teneur en cuivre des arbres échantillonnés.

Tableau II. Récapitulation des cas de carence en cuivre sur résineux.

\begin{tabular}{|c|c|c|c|}
\hline Espèces & Sols & Localisation & Auteurs \\
\hline $\begin{array}{l}\text { Picea sitchensis } \\
\text { Pinus radiata } \\
\text { Picea sitchensis } \\
\text { Pseudotsuga menziesii } \\
\text { Larix sp } \\
\text { Pseudotsuga menziesii } \\
\text { Picea sitchensis } \\
\text { Pinus radiata } \\
\text { Picea sitchensis } \\
\text { Pseudotsuga menziesii } \\
\text { Pinus radiata } \\
\text { Pinus radiata } \\
\text { Pinus cembra } \\
\text { Pinus elliotti } \\
\text { Pseudotsuga menziesii } \\
\text { Picea pungens, Abies procera } \\
\text { Pseudotsuga menziesii } \\
\text { Pinus strobus } \\
\text { Pinus radiata } \\
\text { Pinus radiata } \\
\text { Pinus radiata } \\
\text { Pinus radiata }\end{array}$ & $\begin{array}{l}\text { Sableux acides } \\
\text { Sableux acides } \\
\text { Sols à humus brut } \\
\text { - } \\
\text { Podzol humique et tourbe } \\
\text { Tourbe a Sphaigne } \\
\text { Sols sableux profonds } \\
\text { Sols granitiques } \\
\text { Sols sableux } \\
\text { Sable + terre rouge } \\
\text { Sable fin, acide } \\
\text { Sol podzolique } \\
\text { Sol brun acide, podzol } \\
\text { Tourbe + vermiculite } \\
\text { Roche métamorphique } \\
\text { très dégradée } \\
\text { Sols granitiques } \\
\text { Sols podzoliques } \\
\text { Sable, tourbe sur podzol }\end{array}$ & $\begin{array}{l}\text { Gde-Bretagne } \\
\text { Australie } \\
\text { - } \\
\text { Pays-Bas } \\
\text { Pays-Bas } \\
\text { Scandinavie } \\
\text { Australie SE } \\
\text { Fr Bretagne } \\
\text { Massif Central } \\
\text { Nouv Zélande } \\
\text { Australie Sud } \\
\text { Autriche } \\
\text { Fr Morvan } \\
\text { USA }\end{array}$ & $\begin{array}{l}\text { Benzian et Warren, } 1956 \\
\text { Hall, } 1961 \\
\text { Materna, } 1962 \\
\text { Van Goor, } 1963,1965 \text { a et b } \\
\text { Penningsfeld, } 1964 \\
\text { Oldenkamp et Smilde, } 1966 \\
\text { Haveraaen, } 1967 \\
\text { Ruiter, } 1969 \\
\text { Bonneau, } 1971 \\
\text { Strullu et Bonneau, } 1978 \\
\text { Will, } 1972 \\
\text { Raupach et al, } 1978 \\
\text { Neuwinger et Schinner, } 1980 \\
\text { Hill et al, } 1981 \\
\text { Laffont, } 1981 \\
\text { Lambert et Weidensaul, } 1982 \\
\text { Gonzalez et al, } 1983 \\
\text { Pederich et al, } 1984 \\
\text { Turvey, } 1984 \\
\text { Hunter, } 1987\end{array}$ \\
\hline
\end{tabular}


Des exemples plus récents de carence en cuivre sont mentionnés dans le tableau II, elles sont en général bien corrigées par des pulvérisations foliaires ou des fertilisations appropriées (sulfate de cuivre). On constate que les carences en cuivre sont principalement liées, soit à des sols très pauvres (sables), soit à des sols très riches en matière organique stable (tourbes). Laffont (1981) a pu établir que, dans les sols granitiques du Morvan, l'analyse du Cu total dans l'horizon $A_{1}$ du sol pouvait fournir un bon diagnostic du risque de carence $(<3,5 \mathrm{mg} / \mathrm{kg}$ : risque très élevén; $>9 \mathrm{mg} / \mathrm{kg}$ : risque très faible). Par ailleurs, la tendance à la carence en cuivre sur le pin maritime des Landes est largement renforcée par l'amélioration de la fertilisation phosphatée (Saur, 1989).

le tableau III résume les seuils de carence et les teneurs optimales en cuivre, recensés par Bonneau (1986), dans les aiguilles de differentes espèces résineuses.

Les symptômes de la carence en cuivre des conifères concernent la croissance des axes:

- port buissonnant et pleureur ;

- multiplication du nombre de rameaux par verticille ;
- pousses longues, souples et sinueuses ;

- nécrose du bourgeon terminal.

Certains symptômes sont plus spécifiques:

- apparition de poches de résine dans l'écorce (Pseudotsuga menziensii) ;

- chute précoce des aiguilles (Picea sitchensis) ;

- jaunissement de l'extrémité des ajguilles (Pinus sylvestris).

Les cas de toxicité en cuivre sont rares chez les essences forestières car elles reçoivent en général peu de traitements phytosanitaires a base de sels de cuivre. Des études ont néanmoins permis de mettre en évidence dans les aiguilles des teneurs toxiques pour le végétal : $15 \mathrm{mg} / \mathrm{kg}$ pour Pinus elliotti (Van Lear et Smith, 1972) et $97 \mathrm{mg} / \mathrm{kg}$ pour Picea sitchensis (Burton et Morgan, 1983). Au Kenya, Gibson (1958) signale des dégâts dans les pépinières forestières de Pinus patula par suite de l'utilisation de préparations à base d'oxyde de cuivre pour lutter contre la fonte des semis; ces dégâts se manifestent en sols acides et sont attribues à la toxicité du cuivre qui altère fortement les systemes radiculaires. Dykeman et de Sousa (1966) observent des

Tableau III. Seuils de carence et teneurs optimales en cuivre (mg/kg MS) des feuilles de differentes essences - (Bonneau, 1986).

\begin{tabular}{|c|c|c|c|}
\hline Espèces & $\begin{array}{c}\text { Seuils } \\
\text { carence }\end{array}$ & $\begin{array}{c}\text { Teneur } \\
\text { optimales }\end{array}$ & Auteurs \\
\hline $\begin{array}{l}\text { Picea sitchensis (plantation) } \\
\text { Picea sitchensis (plantation) } \\
\text { Pinus caribeae hondurensis ( } 3 \text { ans) } \\
\text { Pinus radiata } \\
\text { Pinus radiata } \\
\text { Pinus radiata } \\
\text { Pseudotsuga menziesii (plantation) } \\
\text { Pseudotsuga menziesii (plantation) }\end{array}$ & $\begin{array}{c}3 \\
2,8 \\
2,2 \\
1 \\
2 \mathrm{à} 3 \\
2 \\
2,6 \\
4\end{array}$ & $8-11$ & $\begin{array}{l}\text { Strullu et Bonneau, } 1978 \\
\text { Benzian et Warren, } 1956 \text { in Morrison } \\
\text { Bevege, } 1978 \\
\text { Ruiter, } 1969 \text { in Morrison } \\
\text { Woods, } 1983 \\
\text { Will, } 1978 \\
\text { Oldenkamp et Smilde in Morrison } \\
\text { Strullu et Bonneau, } 1978\end{array}$ \\
\hline
\end{tabular}


Tableau IV. Teneurs en manganèse $(\mathrm{mg} / \mathrm{kg} \mathrm{MS})$ des feuilles de différentes espèces résineuses (Bonneau, 1986).

\begin{tabular}{|c|c|c|c|c|}
\hline Especes & $\begin{array}{c}\text { Seuil } \\
\text { carence }\end{array}$ & $\begin{array}{l}\text { Niveau } \\
\text { critique }\end{array}$ & $\begin{array}{l}\text { Teneur } \\
\text { optimale }\end{array}$ & Auteurs \\
\hline $\begin{array}{l}\text { Picea abies } \\
\text { Picea abies } \\
\text { Pinus pinaster } \\
\text { Pinus radiata } \\
\text { Pinus radiata }\end{array}$ & $\begin{array}{c}4-15 \\
20 \\
10 \\
20-30\end{array}$ & 20 & $\begin{array}{l}20 \\
70 \\
20\end{array}$ & $\begin{array}{l}\text { Ingestad, } 1958 \text { in Morrison (1974) } \\
\text { Kreutzer, } 1972 \\
\text { Bonneau et al, } 1968 \\
\text { Will, } 1978 \\
\text { Woods, } 1983\end{array}$ \\
\hline
\end{tabular}

symptômes semblables à ceux de la chlorose ferrique sur Picea mariana, Abies balsamea et Larix laricina, attribués à une toxicité du cuivre dans un sable contenant 3500 à 6700 mg $\mathrm{Cu} / \mathrm{kg}$.

\section{Le manganèse}

Le manganèse est l'un des oligo-éléments les plus importants pour la croissance des végétaux ligneux, dans lesquels il est présent en grande quantité et plus particulièrement chez les conifères.

Les cas de carence en forêt sont rares mais bien identifies sur des sols calcaires et des sols tourbeux très pauvres. Dans un sol marécageux calcaire, Ingestad (1958) a observé une chlorose sur Pinus sylvestris, Picea abies, due à une carence en manganèse. Will (1978) en Nouvelle-Zélande et Woods (1983) en Australie ont décrit cette carence sur Pinus radiata.

Chez les résineux, la carence se traduit par un blanchissement ou un jaunissement de la pointe des aiguilles les plus jeunes et le phénomène progresse du bas vers le haut de la couronne. Dans le tableau IV (Bonneau, 1986), sont mentionnées quelques teneurs optimales ou correpondant à des carences chez les conifères. D'une manière générale, la carence en manganèse fait partie du syndrome classique de la chlorose calcaire sur épicéa (Le Tacon, 1976).

La toxicité manganique en forêt a souvent été suspectée mais rarement démontrée sur le terrain. De plus, les espèces forestières, et surtout les conifères, tolèrent des teneurs élevées en manganèse dans les feuilles ( 1000 $5000 \mathrm{mg} / \mathrm{kg}$ : Stone, 1968). Ces fortes concentrations pourraient traduire une adaptation physiologique à l'excès du métal. Une partie au moins des échecs de régénération de sapins dans les mulls des Vosges a été attribuée à une toxicité manganique (Rousseau, 1960) mais cette hypothèse a été contestée par Drapier (1983).

\section{Le zinc}

Les exemples les plus spectaculaires de l'effet des oligo-élements sur les arbres forestiers sont les carences en zinc sur Pinus ( $P$ radiata, $P$ pinaster), décrites par Stoate (1950) en Australie et sur Pinus kesiya du Mangoro à Madagascar (Rampanana, 1984). A l'exception de ces sols australiens très 
Tableau V. Teneurs en zinc ( $\mathrm{mg} / \mathrm{kg} \mathrm{MS}$ ) des feuilles de différentes espèces de résineux (Bonneau, 1986)

\begin{tabular}{|c|c|c|c|c|}
\hline Especes & $\begin{array}{c}\text { Seuils } \\
\text { carence }\end{array}$ & $\begin{array}{c}\text { Niveau } \\
\text { critiq }\end{array}$ & $\begin{array}{l}\text { Teneur } \\
\text { optim }\end{array}$ & Auteurs \\
\hline $\begin{array}{l}\text { Picea abies } \\
\text { picea sitchensis (solution nutritive) } \\
\text { Pinus caribaea hondurensis ( } 3 \text { ans) } \\
\text { Pinus caribaea hondurensis ( } 30 \text { ans) } \\
\text { Pinus kesiya ( } 15 \text { ans) } \\
\text { Pinus radiata (semis) } \\
\text { Pinus radiata (semis) } \\
\text { Pinus radiata (semis) } \\
\text { Pinus radiata }\end{array}$ & $\begin{array}{c}5 \text { à } 9 \\
4 \\
5 \\
6 \\
1 \text { à } 12 \\
10 \\
1-5\end{array}$ & 15 & $\begin{array}{l}20 \\
19\end{array}$ & $\begin{array}{l}\text { Ferraz et Zöttl, } 1979 \\
\text { Burton et Morgan, } 1983 \\
\text { Bevege, } 1978 \\
\text { Rance et al, } 1982 \\
\text { Rampanana, } 1984 \\
\text { Knight, } 1976 \\
\text { Woods, } 1983 \\
\text { Will, } 1978 \\
\text { Stoate, } 1950 \text { in Morrison (1974) }\end{array}$ \\
\hline
\end{tabular}

pauvres, les carences sont rares et affectent surtout les semis; elles sont favorisées par un ensoleillement important (Viets, 1966). Les signes les plus courants sont la couleur jaune ou bronzée des jeunes feuilles à l'extrémité des branches et la perte précoce des aiguilles âgées; l'allongement des aiguilles est freiné, ce qui produit des rosettes très courtes caractéristiques.

Les seuils de carence ne sont connus que pour quelques pins (tableau V). En tout état de cause, les symptômes de cette affection sont suffisamment marqués et la réponse aux pulvérisations foliaires assez nette pour que le diagnostic ne pose pas de problème.

Les cas de toxicité du zinc sont inconnus en milieu forestier.

\section{Le bore}

La carence en bore semble être la plus commune des carences en oligo-éléments des arbres forestiers. Les principaux cas relevés pour les conifères sont cités dans le tableau VI.

La carence en bore est souvent liée à la pauvreté de la roche-mère en cet élément. D'après Bradford (1966), les cas les plus courants sont observés sur les substrats suivants :

- sols acides d'origine volcanique ou provenant de sédiments fluviatiles;

- sols acides fortement lessivés ;

- sables pauvres en argile et limons;

- tourbes acides;

- sols riches en calcaire actif.

L'apparition des symptômes de carence est aggravée par l'hydromorphie et la concurrence herbacée.

Les déficiences observées chez les conifères pour des teneurs foliaires inférieures à $10 \mathrm{mg} \mathrm{B} / \mathrm{kg}$ (tableau VII) provoquent la nécrose des bourgeons terminaux pendant la période d'élongation, le développement d'aiguilles courtes et tordues virant au jaune et au brun et, dans certains cas, l'exsudation de résine à travers l'écorce. Dans le cas de subcarence, on observe une croissance sinueuse des axes, une perte de la dominance apicale et un raccourcissement des entre-nœuds distaux des pousses.

Les cas de toxicité sont surtout connus en pépinière après des traitements du sol contre la carence, ils sont donc très rares. Ils ont été décrits par Stone et Baird (1956) sur Pinus resinosa et Pinus strobus, en dispositif expérimental. 
Tableau VI. Revue bibliographique des carences en bore constatées sur des résineux.

\begin{tabular}{|c|c|c|c|}
\hline Espèces & Sols & Localisation & Auteurs \\
\hline $\begin{array}{l}\text { Pinus patula } \\
\text { Pinus radiata, } P \text { pinaster } \\
\text { Pinus radiata, P pinaster } \\
\text { Pinus radiata, } P \text { patula } \\
\text { Pinus spp } \\
\text { Pinus radiata, P pinaster } \\
\text { Pinus spp } \\
\text { Pinus radiata } \\
\text { Pinus patula } \\
\text { Cupressus lusitanica } \\
\text { Pinus sylvestris } \\
\text { Picea abies, Pinus } \\
\text { sylvestris, } P \text { contorta } \\
\text { Pinus sylvestris } \\
\text { Pinus taeda, } P \text { elliotti } \\
\text { Pinus radiata } \\
\text { Pinus sylvestris } \\
\text { Pinus sylvestris } \\
\text { Picea abies } \\
\text { Pinus radiata }\end{array}$ & $\begin{array}{l}\text { Limons granitiques } \\
\text { Limons, sables, acides } \\
\text { Roches basaltiques } \\
- \\
- \\
- \\
- \\
- \\
\text { Sol tourbeux } \\
\text { Sol tourbeux } \\
\text { Sol tourbeux } \\
\text { Sol volcanique } \\
\text { Sol tourbeux } \\
\text { Sol minéral } \\
\text { Limon sableux } \\
\text { (granodiorite) }\end{array}$ & $\begin{array}{l}\text { Rhodésie } \\
\text { Nouv Zélande } \\
\text { Nouv Zélande } \\
\text { Tanzanie } \\
\text { Brésil } \\
\text { Chili } \\
\text { Malaisie } \\
\text { Australie } \\
\text { Colombie } \\
\text { Finlande } \\
\text { Norvège } \\
\text { Finlande } \\
\text { Floride } \\
\text { Australie } \\
\text { Suède } \\
\text { Suède } \\
\text { Australie }\end{array}$ & $\begin{array}{l}\text { Vail et al, } 1961 \\
\text { Will et al, } 1963 \\
\text { Stone et Will, } 1965 \\
\text { Procter, } 1967 \\
\text { Van Goor, } 1965 \text { a } \\
\text { Tollenaar, } 1969 \\
\text { Waring, } 1971 \\
\text { Snowdon, } 1973 \\
\text { Ladrach, } 1978 \\
\text { Silfverberg, } 1979 \\
\text { Braekke, } 1979 \\
\text { Raitio, } 1979 \\
\text { Stone et al, } 1982 \\
\text { Snowdon, } 1982 \\
\text { Möller, } 1983 \\
\text { Aronsson, } 1983 \\
\text { Hopmans et Flinn, } 1984\end{array}$ \\
\hline
\end{tabular}

Tableau VII. Teneurs en bore ( $\mathrm{mg} / \mathrm{kg}$ MS) des feuilles de différentes espèces de résineux (Bonneau, 1986).

\begin{tabular}{|c|c|c|c|c|}
\hline Espèces & $\begin{array}{c}\text { Seuils } \\
\text { carence }\end{array}$ & $\begin{array}{l}\text { Niveau } \\
\text { critique }\end{array}$ & $\begin{array}{c}\text { Teneur } \\
\text { optimale }\end{array}$ & Auteurs \\
\hline $\begin{array}{l}\text { Picea abies } \\
\text { Picea abies (plantation) } \\
\text { Picea abies (adulte sur sol minéral) } \\
\text { Picea abies (adulte sur sol organique) } \\
\text { Picea abies (adulte) } \\
\text { Picea sitchensis (semis) } \\
\text { Pinus contorta (plantation) } \\
\text { Pinus pinaster } \\
\text { Pinus radiata } \\
\text { Pinus radiata } \\
\text { Pinus radiata } \\
\text { Pinus radiata } \\
\text { Pinus silvestris (plantation) } \\
\text { Pinus silvestris (adulte, sol minéral) } \\
\text { Pinus silvestris (adulte, sol organique) } \\
\text { Pseudotsuga menziesii }\end{array}$ & $\begin{array}{c}8-12 \\
8 \\
3 \\
3 \\
\\
3 \\
6-8\end{array}$ & $\begin{array}{c}13 \\
8-10\end{array}$ & $\begin{array}{c}20-45 \\
20-45 \\
8-12 \\
\\
25-30 \\
16 \\
12\end{array}$ & $\begin{array}{l}\text { Ferraz et Zöttl, } 1979 \\
\text { Braekke, } 1979 \\
\text { Fiedler et Höhne, } 1984 \\
\text { Fiedler et Höhne, } 1984 \\
\text { Silfverberg, } 1980 \\
\text { Gessel et al, } 1979 \\
\text { Braekke, } 1979 \\
\text { Stone et Will, 1965, in Morrison (1974) } \\
\text { Will, 1978, Miller, 1981 } \\
\text { Hopmans et Flinn, } 1984 \\
\text { Miller, 1981 } \\
\text { Will, 1972 } \\
\text { Braekke, 1979 } \\
\text { Fiedler et Höhne, } 1984 \\
\text { Fiedler et Höhne, } 1984 \\
\text { Carter et al, 1984 }\end{array}$ \\
\hline
\end{tabular}




\section{CONCLUSION}

La sylviculture est un domaine où l'on n'aborde que sporadiquement la nutrition oligo-minérale. Les problèmes de toxicité connus dans les cas de pollution des cultures agricoles sont très rares en forêt, où les modifications anthropiques de nature chimique sont encore faibles.

Les situations de carence sont connues et traitées lorsque leur influence sur la production de biomasse est très marquée et les interventions sylvicoles rentables. Ces carences sont généralement liées à la pauvreté originelle de la roche-mère et aux conditions physico-chimiques des sols $(\mathrm{pH}$ extrêmes, anoxie, etc).

Les connaissances fondamentales sur la nutrition oligo-minérale sont développées pour les plantes de grande culture mais restent réduites pour les espèces ligneuses. Or, il est admis que de nombreux résultats obtenus pour des plantes annuelles ne sont pas transposables aux plantes pérennes. On peu raisonnablement espérer de nombreux progrès en sylviculture (choix des espèces, diagnostic et correction de subcarences ou de déséquilibres nutritionnels) par une meilleure connaissance des phénomènes physiologiques d'absorption racinaire propres aux arbres et une bonne compréhension des mouvements d'éléments entres les differents tissus.

En effet, l'étude de la dynamique des oligo-éléments dans un système aussi complexe que celui de l'arbre doit permettre de définir des "pools" actifs pour les tissus vivants, dans le cadre d'une croissance cyclique accompagnée de phénomènes de mise en réserve et de remobilisation.

\section{RÉFÉRENCES}

Ahrens $E$ (1965) Untersuchungen über den, Cu-, $\mathrm{Zn}$-, B- und Mo-gehalt verschiedener Baumarten. Vortr Bodenk Coll, Münden, Han

Amberger A (1973) The role of Manganese in the metabolism of plants. Agrochimica, $17,69-83$

Aronsson A (1983) Growth disturbances caused by Boron deficiency in some fertilized pine and spruce stands on mineral soils. Comm Inst For Fenn, 116, 116-122

Aubert H, Pinta M (1971) Les élémentstraces dans les sols. ORSTOM travaux \& Doc $n^{\circ} 11,104 \mathrm{pp}$

Benzian B, Warren RG (1956) Copper deficiency in Sitka spruce seedlings. Nature, 178, 864-865

Bevege B (1978) Foliar analysis as a guide to the nutrient status of tropical pines. In PI Nutr: Proc $8^{\text {th }}$ Coll on Plant analysis and fertilizer problems, Aukland (NZ) (AR Fergusson, RL Bieleski, TB Fergusson, eds) $1972 / 09 / 01$

Bingham FT (1963) Relation between phosphorus and micronutrients in plants. Soil Sci Soc Am Proc, 27, 389-391

Bingham FT, Garber MJ (1960) Solubility and availability of micronutrients in relation to phosphorus fertilization. Soil Sci Soc Am Proc, 24, 209-213

Bingham FT, Martin JP, Chastain JA (1958) Effects of phosphorus fertilization of California soils on minor element nutrition of Citrus. Soil Sci, 86, 24-31

Bingham FT, Elseewi A, Oertli JJ (1970) Characteristics of Boron absorption by excised barley roots. Soil Sci Soc Am Proc, 34, 613-617

Bonneau M (1971) Causes de la deformation des jeunes Douglas dans le Limousin. Ann Sci For, 28, 341-353

Bonneau M (1986) Cours de Pédagogie forestiere. Diagnostic foliaire. ENGREF, Nancy, $58 \mathrm{pp}$

Bonneau M, Gelpe J, Le Tacon F (1968) Influence des conditions de nutrition minérale sur le dépérissement du Pin maritime dans les Landes de Gascogne. Ann Sci For, 25, 251-289

Bowen JE (1969) Absorption of Copper, Zinc and Manganese by sugar cane tissue. Plant Physiol 44, 255-261 
Bowen JE, Nissen P (1976) Boron uptake by excised barley roots. I. Uptake into the free space. Plant Physiol, 57, 353-357

Bradford GR (1966) Boron In: Diagnostic criteria for plants and soils, Univ California, 4, 33-61

Braekke FH (1979) Boron deficiency in forest plantations on peat land in Norway. Medd Nor inst Skogforsk, 35, 213-236

Bremmer I, Knight AH (1970) The complexes of Zinc, Copper and Manganese present in Rye-grass. Brit $J$, Nutr, 24, 279-290

Burton KW, Morgan E (1983) The influence of heavy metals upon the growth of Sitka spruce in South Wales forests. I. Upper critical foliar concentration. Plant Soil, 73 , 327-336

Cannel GH, Bingham FT, Garber MJ (1960) Effects of irrigation and phosphorus on vegetative growth and nutrient composition of Tomato leaves. Soil Sci, 89, 53-60

Carter RE, Otchere-Boateng J, Klinka K (1984), Dieback of a 30 year-old Douglas fir plantation in the Britain River Valley, British Columbia: symptoms and diagnosis. For Ecol Manage, 4, 249-263

Cathala DA, Salsac L (1975) Absorption du cuivre par les racines de mais et de tournesol. Plant Soil, 42, 65-83

Chaudhry FN, Loneragan JF (1972) Zinc absorption by wheat seedlings. Soil Sci Soc Am Proc, 36, 323-331

Clarkson DT, Hanson JB (1980) The mineral nutrition of higher plants. Ann Rev Plant Physiol, 31, 239-298

Colozzi-Filho A, Siqueira JO (1986) Vesicular arbuscular mycorrhiza in coffee seedlings. I. Effects of Giga spora margarita and phosphate additions on plant growth and nutrition. Rev Bras Ciencia Solo, 10. 199-205

Coppenet M, Jolivet E (1952) Détection de graves carences en cuivre dans les terres très humifères du centre de la Bretagne. CR Acad Agric Fr, 38, 691-693

Datta M, Gupta RK (1984) Effect of superphosphate on the yield of Wheat and Rice on acid soil of Nagaland and nutrient content of grain. J Indian Soc Soil Sci, 32, 299-302

Dekock PC, Cheshire MV, Hall A (1971) Comparison of the effects of phosphorus and nitrogen on $\mathrm{Cu}$ deficient and suffering oats. J Sci Food Agric, 22, 431-440
Delas J (1967) Liaisons entre cuivre et matière organique dans un podzol développé sur sable des Landes et accidentellement enrichi en cuivre. Ann Agron, 18, 17-29

Dokiya Y, Kumazawa K, Mitsui S (1964) Nutrient uptake of crop plants. 4. The comparative physiological study on the uptake of Iron, Manganese and Copper by plants. The uptake of $58^{\mathrm{Fe}}, 54^{\mathrm{Mn}}, 64^{\mathrm{Cu}}$ by rice and barley seedling as influenced by metabolic inhibitors. J. Sci Soil Manure, Japan, 35, 367-378

Dolar SG, Keeney DR (1971) Availability of $\mathrm{Cu}, \mathrm{Zn}$ and $\mathrm{Mn}$ in soils. I. Influence of soil $\mathrm{pH}$, organic matter and extractable phosphorus. J Sci Food Agric, 22, 273282

Downes G, Turvey ND (1986) Reduced lignification in Pinus radiata D Don Austr For Res, 16, 317-377

Drapier J (1983) Les difficultés de régénération des sapinières vosgiennes. Importance de l'humus et rôle de l'allèlopathie. Thèse Univ Nancy I, 109 pp

Drouineau G, Mazoyer R (1962) Contribution à l'étude de la toxicité du cuivre dans les sols. Ann Agron, 13, 31-53

Duval L (1963) Existence de graves carences en cuivre dans les sols gréseux de la Basse-Normandie et du Bas-Maine. $C R$ Acad Agric $F r, 49,1216-1220$

Duval L, Maurice J (1970) Le diagnostic des carences en oligo-éléments au moyen de l'analyse chimique des sols. Ann Agron, $21,573-586$

Dykeman WR, de Sousa AS (1966) Natural mechanism of Copper tolerance in a Copper swamp forest. Can J Bot, 44, 871-878

Elgawhary SM, Lindsay WL, Kemper WD (1970) Effect of complexing agents and acids on the diffusion of Zinc to a simulated root. Soil Sci Soc Am Proc, 34, 211-219

Ferraz J, Zöttl HW (1979) Nährelment spiegel und Spurelementnerteilung in Fichtenbestanden des GrundgebirgerSchwarzwaldes. Mitt Dtsch Bodenk Gesellsch 29, 587-594

Fiedler HJ, Höhne H (1984) Die Bor-Ernährung von koniferen und ihre beziehung zum gehalt and calcium und kalium in den assimilationsorganen. Beitr Forstwirtsch, 18, 73-80 
Gessel SP, Steinbrenner EC, Miller RE (1979) Response of Northwest forests to element other than nitrogen. In : Proc Forest Fertilization Conf (SP Gessel, Kenady RM, Alkinson WA, eds) Univ Washington, Coll For Resources, Inst For Resources. Contribution 40, 140-149

Gibson IAS (1958) Phytotoxic effects of Copper fongicids on acid soils. East Afr Agric J. 24, 125-127

Gildon A (1983) The relationship of Vesicular-arbuscular mycorrhizal infection and Copper nutrition. J Sci Food Agric, 34. 56-57

Gildon A, Tinker PB (1983) Interactions of Vesicular arbuscular mycorrhizal infection and heavy metals in plants. I. The effect of heavy metals on the development of Vesicular arbuscular mycorrhizas. New Phytol, 95, 247-261

Giordano PM, Noggle JC, Mortvedt JJ (1974) Zinc uptake by rice as affected by metabolic inhibitors and competing cations. Plant Soil, 41, 637-646

Godo GH, Reisenauer HM (1980) Plant effects on soil Manganese availability. Soil Sci Soc Am J, 44, 993-995

Gonzalez C. Konow V, Lachica M, (1983) El Pinus radiata $D$ Don en Chile. Ensayos de fertilización con cobre y boro. An Edafol Agrobiol, 42, 1599-1613

Graham JH, Timmer LW, Fardelmann D (1986) Toxicity of fungicidal Copper in soil to Citrus seedlings and Vesicular arbuscular mycorrhizal fungi. Phytopathology, 76, 66-70

Greenwood EAH, Hallsworth EG (1960) Studies on the nutrition of forage legumes. II. Some interactions of Calcium, Phosphorus, Copper and Molybdenum on the growth and chemical composition of Trifolium subterraneum L. Plant Soil, 12, 97-127.

Guillet B, Jeanroy E, Rougier RC, Souchier B (1980) Le cycle biogéochimique et la dynamique du comportement des éléments-traces ( $\mathrm{Cu}, \mathrm{Pb}, \mathrm{Zn}, \mathrm{Ni}, \mathrm{Co}, \mathrm{Cr}$ ) dans les pédogenèses organiques acides. Rapport ATP «Formation et distribution des gisements". Note tech sci centre de pédologie, Nancy, 27, 49

Hall IR, Johnstone PD, Dolby R (1984) Interactions between endomycorrhizes and soil Nitrogen and Phosphorus on the gowth of Rye-Grass. New Phytol, 97, 447-453
Hall JR (1972) The effect of Phosphorus, season and method of sampling on foliar analysis, mineral depletion and yield of Merion Kentucky blue grass. Dissert Abst Int, B Sci Eng, 32, 3739-3740

Hall MJ (1961) The establishment of pines on poor deep sands at Longford, Victoria. Appita, 15, 68-76

Halstead EH, Barber SA, Warncke DO, Bole J (1968) Supply of $\mathrm{Ca}, \mathrm{Sr}, \mathrm{Mn}$ and $\mathrm{Zn}$ to plant roots. Soil Sci Soc Am Proc, 32, 69-72

Harrison SJ, Lepp NW, phipps DA (1979) Uptake of Copper by excised roots. II. Copper desorption from the free space. $Z$ Pflanzenernaehr Bodenkde, 94, 27-34

Haveraaen SO (1967) Copper deficiency on Norway Spruce. Medd Nor Inst Skogforsk, 23, 289-300

Hill JM (1973) The changes with age in the distribution of Copper and some Coppercontaining oxidases in red clover (Trifolium pratense $\mathrm{L}$ c $v$ Dorset Marlgrass). $J$ Exp Bot, 24, 525-536

Hill JM, Turvey ND, Bevege DI (1981) Copper deficiency of Pinus $s p$ in Eastern Australia. In : Copper in soils and plants (JF Loneragan et al eds) Acad Press, Sydney, $362 \mathrm{pp}$

Hodgson JF (1963) Chemistry of the micronutrient elements in soils. Adv Agron, 15, 119-159

Hodgson JF, Lindsay WL, Trierweiler JF (1966) Micronutrient cation complexing in soil solution. II. Complexing of Zinc and Copper in displacing solution from calcareous soils. Soil Sci Soc Am Proc, 30, 723-726

Hopmans P, Flinn DW (1984) Boron deficiency in Pinus radiata $D$ Don and the effect of applied Boron on height growth and nutrient uptake. Plant Soil, 79, 295-298

Hulagur BF, Dangarwala RT, Mehta BV (1975) Effect of Zinc, Copper and Phosphorus on the yield and composition of hybrid Maize grown in loamy sand. $J$ indian Soc Soil Sci, 23, 83-90

Hunter IR (1987) Field experience with Copper fertilizer in New-Zealand. In : Workshop on $B, C u, Z n$ nutrition in plantation forestry, Rotura (New Zeal), 1987/10/01-07

Ingestad J (1958) Studies on Manganese deficiency in a forest stand. Medd Statens Inst Skogforsk, 48, 3-20 
Jarvis SC (1978) Copper uptake and accumulation by perennial Ryegrass grown in soil and solution culture. J Sci Food Agric, 29, 12-18

Jones JB Jr (1970) Distribution of 15 elements in corn leaves. Commun Soil Sci Plant Anal, 1, 27-34

Juste C (1988) Appréciation de la mobilité et de la biodisponibilité des éléments en traces du sol. Sci Sol, 26, 103-112

Kabata-Pendias A, Pendias H (1984) Trace elements in soils and plants. CRC Press, $315 \mathrm{pp}$

Keller P, Deuel H (1958) Cation exchange equilibrium with dead plant roots. Trans Comm II and IV Soc Soil Sci, Verlag Chemic Weinheim Hamburg, 2, 164-168

Knight PJ (1976) Zinc deficiency in nursery grown Pinus radiata seedlings. NZJ For Sci, 5, 260-264

Kovda VA, Yakushevskaya IV, Tyurukanov AN (1964) Micronutrients of the soils in the Union of Soviet Socialist Republics. UNESCO INSINR 149, Paris, Fev 1964

Kraustopf KB (1972) Geochemiotry of micronutrients. In : Micronutrients in Agriculture. Soil Sci Soc Am Madison (USA) 2, 7-40

Kreutzer K (1972) Die wirkung des manganmangels auf die farhe, die pigmente und den gaswechsel von ficthennadeln. Forstwiss Centralbl, 91, 80-98

Krishna KR, Bagyaraj DJ (1984) Growth and nutrient uptake of Peanut inoculated with the mycorrhizal fungus Glomus fasciculatus compared with non-inoculated ones. Plant Soil, 77, 405-408

Kucey RMN (1987) Mycorrhizal fungi increase plant micronutrient uptake. Res Highlights 1985-86, Ottawa Can, Agriculture Canada, 94-95

Labanauskas CK, Embleton TW, Jones WW (1958) Influence of soil applications of Nitrogen, Phosphate, Potash, dolomite and marne on micronutrient content of Avocado leaves. Proc Am Soc Hortic Sci, $71,285-291$

Labanauskas CK, Embleton TW, Jones WW, Garber MJ (1959) Effects of soil applications of Nitrogen, Phosphate, Potash, dolomite and marne on the micronutrient concentration in Valencia Orange leaves. Proc Am Soc Hortic Sci, 73, 257-266
Ladrach WE (1978) Second year results of $\mathrm{N}, \mathrm{P}$ and $\mathrm{B}$ fertilizer application at planting with Cupressus lusitanica, Pinus patula, Eucalyptus grandis . Invest For Colombia, 36

Laffont H (1981) Deformations chez le Douglas (Pseudotsuga menziesii): problème des carences en cuivre et en zinc des peuplements du Morvan granitique. DEA, Univ Nancy I, $48 \mathrm{p}$

Lal R, Taylor GC (1970) Drainage and nutrient effects in a field lysimeter study. II. Mineral uptake by corn. Soil Sci Soc Am Proc, 34, 245-248

Lambert DH, Baker DE, Cole H Jr (1979) The role of mycorrhizae in the interactions of phosphorus with Zinc, Copper and other elements. Soil Sci Soc Am J, 43, 976-980

Lambert DH, Weidensaul TC (1982) Copper requirement of container-grown conifer seedlings. Can J For Res, 12, 848-852

Leep NW (1979) Cycling of Copper in woodland ecosystems. In: Copper in the environment. Part / (JO Nriagh ed) Wiley, New-York, 289-323

Le Tacon F (1976) La présence de calcaire dans le sol, influence sur le comportement de l'épicea commun (Picea excelsa Link) et du Pin noir d'autriche (Pinus nigra nigricans Host). These Doct INPL Nancy, 214 pp

Lipman CB, Mac Kinney G (1931) Proof of the essential nature of Copper for higher green plants. Plant Physiol, 6, 593-599

Löhnis MP (1960) Effect of magnesium and calcium supply on the uptake of Manganese by various crop plants. Plant Soil, $12,339-376$

Loneragan JF (1975) The availability and absorption of trace elements in soil-plant systems and their relation to movement and concentration of trace elements in plants. In: Trace elements in soil-plant-animal systems, Acad Press, London, 109-134

Loneragan JF (1977) Plant efficiencies in the use of $\mathrm{B}, \mathrm{Co}, \mathrm{Cu}, \mathrm{Mn}$ and $\mathrm{Zn}$. In: Plant adaptation to mineral stress in problem soils. Special Publ Cornell Univ, Agric Exp Stn New-York, 193-203

Loneragan JF, Snowball K, Robson AD (1980) Copper supply in relation to content and redistribution of Copper among organs of the wheat plant. Ann Bot (Lond), 45, 621-632 
Loue A (1986) Les oligo-élements en agriculture. Agri-Nathan, $339 \mathrm{pp}$

Lucas RL, Knezek BD (1972) Climatic and soil conditions promoting micronutrient deficiencies in plants. In : Micronutrients in Agriculture, Soil Sci Soc Am, Madison (USA), 12, 265-288

Maas EV, Moore DP, Mason BJ (1968) Manganese absorption by excised barley roots. Plant Physiol, 43, 527-530

Maas EV, Moore DP, Mason BJ (1969) Influence of Calcium and Magnesium on Manganese absorption. Plant Physiol, 44, 796-800

Mac Bride MB, Blasiak JJ (1979) Zinc and Copper solubility as a function of $\mathrm{pH}$ in an acid soil. Soil Sci Soc Am Proc, 43, 866-870

Mac Laren RG, Crawford DV (1973) Studies on soil Copper. 1. The fractionation of $\mathrm{Cu}$ in soils. $J$ Soil Sci, 24, 172-181

Mc Hargue JS (1925) Occurence of Copper, Manganese, Zinc, Nickel and Cobalt in soils, plants and animals, and their possible function as vital factors. $J$ Agr Res, 30, 193-196

Massey HF, Loeffel A (1967) Species specific variations in Zinc content of corn kernels. Agron J, 214-217

Materna J (1962) Kupfer, Zink und Mangangehalte in Fichtenbestanden. Tagunsber Deut Akad Land Wiss, Berlin, 50, 45-52

Meek BD, Mac Kenzie AJ, Grass LB (1968) Effects of organic matter flooding time and temperature on the dissolution of Iron and Manganese in soil. Soil Sci Soc Am Proc, 21, 625-628

Mengel K, Kirkby EA (1982) Principles of plant nutrition. Int Potash Inst, $3^{\Theta}$ édit $655 \mathrm{pp}$

Michael $G$, Wilberg E, Kouhsiahi-Tork K (1969) Durch hohe luftfenchtigkrit induzierter bormangel. Z Pflanzemerachr bodenkde 122, 1-3

Miller HG (1981) Aspects of forest fertilization practice and research in New-Zealand. Scott For, 35, 277-288

Möller G (1983) Variation of Boron concentration in pine needles from trees growing on mineral soil in Sweden and response to nitrogen fertilization. Commun Inst For Fenn, 116, 111-115

Moore DP (1972) Mechanisms of micronutrient uptake by plants. In: Micro- nutrients in : agriculture, Soil Sci Soc Am, Madison (USA), 171-198

Morrison IK (1974) Mineral nutrition of conifers with special reference to nutrient status interpretation: a review of literature. Can For Serv, Ottawa, Ontario, Pub $n^{\circ} 1343$ Dep Envir, $74 \mathrm{pp}$

Muller J (1960) Échange des ions cuivriques sur les colloïdes minéraux. Ann Agron, 75-91

Neuwinger I, Schinner F (1980) The influence of compound fertilizer and cupric sulfate on the gowth and bioelement content of Cembra pine seedlings (Pinus cembra). Plant Soil, 57, 257-270

Nielsen NE (1976) The effect of plants on the Copper concentration of the soil solution. Plant Soil, 45, 679-687

Nissen $P$ (1974) Uptake mecanisms: Inorganic and organic. Ann Rev Plant Physiol, 25, 53-72

Oertli JJ (1963) The influence of certain environmental conditions on water and nutrient uptake and nutrient distribution in barley seedlings with special reference to Boron. Adv Front Plant Sci, New-Delhi, 6 , 55-85

Oldenkamp L, Smilde KW (1966) Copper deficiency in Douglas Pine (Pseudotsuga menziesii, Mirb Franco). Plant Soil, 25, 150-152

Pacovsky RS, Paul EA, Bethlenfalvey GJ (1986a) Comparison between P-fertilized and mycorrhizal plants. Crop Sci, 26, 151-156

Pacovsky RS, Paul EA, Bethlenfalvay GJ (1986b) Response of mycorrhizal and P. fertilized Soybeans to nodulation by Bradyrhizobium or Ammonium nitrate. Crop Sci, 26, 145-150

Parker DR, Gardner EH (1982) Factors affecting the mobility and plant availability of Boron in some Western Oregon soils. Soil Sci Soc Am J, 46, 573-578

Patra D, Haldar M, Mandal LN (1982) Effect of $\mathrm{P}, \mathrm{Cu}$ and $\mathrm{Zn}$ application on the growth and $\mathrm{Zn}, \mathrm{Cu}, \mathrm{Fe}, \mathrm{Mn} \mathrm{P}$ nutrition of Rice in water logged soil. Indian Agriculturist, 26 , 229-235

Pederich LA, Hopmans P, Flinn DW, Abbott ID (1984) Variation in genotypic response to suspected Copper deficiency in Pinus radiata. Aust For Res, 14, 75-84 
Penningsfeld F (1964) Nährstoffmangelersheinungen bei Baumschulgehölzen. Phosphorsäure, 24, 199-212

Procter $J$ (1967) A nutritional disorder of Pine. Commonw. For Rev, 46, 145-154

Qayyum R,-ul, Athar M (1981) Effect of Nitrogen, Phosphorus and Potassium on the uptake pattern of Zinc and Copper in Rice. J Agric Res, Pakistan, 19, 109-115

Rahimi A, Bussler M (1974) Kupfermangel bei höheren pflanzen und sein histochemicher Nachweir. Landwirtsch Forsch, 27, 101-111

Raitio H (1979) Growth disturbances of Scots pine caused by Boron deficiency on an afforested abandoned peatland field. Description and interpretation of symptoms. Folia For (Helsinki), 412, $16 \mathrm{pp}$

Rampanana L (1984) Nutrition minérale du Pinus kesiya dans la zone du Mangoro. Cas particulier du potassium et du zinc. These $3^{\mathrm{e}}$ Cycle, Univ Madagascar, $141 \mathrm{pp}$

Rance SJ, Cameron DM (1982) Correction of crown disorders of Pinus caribaea var hondurensis. Plant Soil, 65, 293-296.

Raupach M, Clarke ARP, Cellier KM (1978) Disorder symptoms of a forest of Pinus radiata in relation to foliar nutrient levels. Aust For Res, 8, 1-11

Raven JA (1980) Short and long distance transport of boric acid in plants. New Phytol, 84, 231-249

Redlich GC (1954) Les problèmes de la culture des sables des Landes. Bull CETA, 15, 149-151

Reuter DJ, Robson AD, Loneragan JF, Trathim-Fryer DJ (1981) Copper nutrition of subterranean clover (Trifolium subterraneum L cv Seaton Park). III. Effects of Phosphorus supply on the relationship between Copper concentration in plant parts and yield. Aust J Agric Res, 32, 283-294

Riceman DS, Jones GB (1958) Distribution of $\mathrm{Zn}$ and $\mathrm{Cu}$ in subterranean clover (Trifolium subterraneum $L$ ) grown in solution cultures supplied with graduated amounts of $\mathrm{Zn}$. Aust J Agric Res, 9, 73-122

Rousseau LZ (1960) De l'influence du type d'humus sur le développement des plantules de sapin dans les Vosges. Ann École Nat Eaux For, 17, $118 \mathrm{pp}$
Ruiter JH (1969) Suspected Copper deficiency in radiata pine. Plant Soil, 31 , 197-200

Russ E (1958) The determination of available Copper and Manganese in soils with particular regard to the seedling method. Diss Landw Fakultăt Giessen

Sandmann G, Böger P (1983) The enzymatological function of heavy metals and their role in electron transfer process of plants. In: Encyclopedia of plant physiology Springer Verlag, 15A, 563-596

Sasa M, Zahka G, Jakobsen I (1987) The effects of pretransplant inoculation with VA mycorrhizal fungi on the subsequent growth of leeks in the field. Plant Soil, 97 , 279-283

Sauchelli V (1969) Trace elements in agriculture. Van Nostrand Reinhold Co, $248 \mathrm{pp}$

Saur E (1989) Effect of phosphate fertilization on trace element nutrition of Pinus pinaster grown in a acid sandy soil. Proc Int Symp For tree physiol, Nancy, 1988. Ann Sci For. Suppl 46, 6905-6930

Schnitzer M., Skinner SIM (1966) Organometallic interactions in soils: V. Stability constants of $\mathrm{Cu}^{2+}, \mathrm{Fe}^{2+}$ and $\mathrm{Zn}^{2+}$ fulvic acid. Soil Sci, 102, 361-366

Schmid WE, Haag HP, Epstein E (1965) Absorption of Zinc by excised barley roots. Physiol Plant, 18, 860-869

Sidle RC, Shaw CG (1987) Évaluation of planting sites commonto a South-east Alaska clear-cut. IV. Nutrient levels in ectomycorrhizal Sitka spruce seedlings. Can J For Res, 17, 340-345

Silfverberg $K$ (1979) Phenology and initial development of a growth disorder in scot pine on Boron deficient peat land. Folia For Helsinki, 396, 1-19

Silfverberg $K$ (1980) A micronutritional growth disorder in Norway Spruce. Folia For Inst For Fenn, 432

Sims JR, Bingham FT (1968) Retention of Boron by layer silicates, sesquioxides and soil material. Soil Sci Soc Am Proc, 32, 364-373

Snowdon $P$ (1973) Problems in the diagnosis of Boron deficiency in Pinus radiata. In : FAO/IUFRO Intern Symp For Fertil, Paris, 1973/12/03-07

Snowdon P (1982) Diagnosis of Boron deficiency in soils by pot experiments with Pinus radiata. Aust For Res, 12, 217-229 
Sommer AL (1931) Copper as an essential for plant growth. Plant Physiol, 6, 339-345

Spencer WF (1960) Effects of heavy applications of phosphate and lime on nutrient uptake, growth freeze of grapefruit trees. Soil Sci, 89, 311-318

Spencer WF (1966) Effect of Copper on yield and uptake of Phosphorus and Iron by Citrus seedlings grown at various Phosphorus levels. Soil Sci, 102, 296-299

Stoate TN (1950) Nutrition of the Pine. Aust For Timber Bur, Bull 30, 61

Stone EL (1968) Micro-element nutrition of forest trees: A review. In : Forest fertilization, Theory and Practice, Tennessee Valley Authority, Alabama, 132-175

Stone EL, Baird G (1956) Boron level and Boron toxicity in red and white Pine. J For, $54,11-12$

Stone EL, Will GM (1965) Boron deficiency in Pinus radiata and Pinaster. For, Sci, 11, 425-433

Stone EL, Hollis CA, Barnard EL (1982) Boron efficiency in a Southern Pine nursery. South J App/ For, 6, 108-112

Strullu DG, Bonneau M (1978) Contribution à l'étude des carences en cuivre chez les Abietacées. J Can Bot, 56, 2648-2659

Swaine DJ (1955) The trace element content of soil. Commonw Bur Sci Tech, 48 pp

Tanaka H (1967) Boron adsorption by plant roots. Plant Soil, 27, 300-302

Thellier M, Duval Y, Demarty M (1979) Borate exchanges of Lemua minor $L$ as studied with the help of the enriched stable isotope and of a $(n, \alpha)$ nuclear reaction. Plant Physiol, 63, 283-288

Tiffin LO (1967) Translocation of manganese, Iron, Cobalt and Zinc in Tomato. Plant Physiol, 42, 1427-1432

Tiffin LO (1972) Translocation of micronutrients in plants. In: Micronutrients in Agriculture (JJ Mortvedt, PM Giordano, WL Lindsay, eds) Soil Sci Soc Am Inc, Madison, W!, 199-229

Tiffin LO (1977) The form and distribution of metals in plants: an overview. Proc Hanford Life Sci Symp US Dept Energy, Symp Series, Washington DC, 315

Timmer LW, Leyden RF (1980) The relationship of mycorrhizal infection to Phosphorus-induced Copper deficiency in Sour orange seedlings. New Phytol, 85, $15-23$
Tinker PB (1981) Levels, distribution and chemical forms of trace elements in food plants. Philos Trans R Soc, London, 249, 41-47

Tollenaar H (1969) Boron deficiency in Pine plantations in the Central zone of Chile. Agric Tec (Santiago), 29, 85-88

Turvey ND (1984) Copper deficiency in Pinus radiata planted in a podzol in Victoria, Australia. Plant Soil, 77, 73-86

Vail JW, Parry MS, Calton WE (1961) Boron deficiency dieback in pinus. Plant Soil, 14, 393-398

Van Goor BJ (1974) Distribution of mineral nutrients in the plant in relation to physiological disorder. Proc $19^{\text {th }}$ Int Hor Congr Warsaw, 1974/09/11, 217

Van Goor BJ, Wiersma D (1976) Chemical form of Manganese and Zinc in phloem exudates. Physiol Plant, 36, 213-219

Van Goor CP (1963) Fertilization of conifer plantations. Korte Meded Sticht BosbProefsta, 56, 129-142

Van Goor CP (1965a) Reflorestamento com coniferas no Brazil. Ministerio de Agricultura, Servico Florestal, Setor Inv FI Bol, 58

Van Goor CP (1965b) Spurelemente bei der Ernahrung von Koniferen. Vorrag Anl Tag Arbeitsgern Forst Tharandt, 10, 4-6

Van Lear DH, Smith WH (1972) Relationships between macro- and micronutrient nutrition of slash pine on three coastal plain soils. Plant Soil, 36, 331-342

Vaughan D, Dekock PC, Ord BG (1982) The nature and localization of superoxide dismutase in fronds of Lemna gibba $\mathrm{L}$ and the effect of copper and zinc deficiency on its activity. Physiol Plant, 54, 253-257

Verlière $G$ (1973) La nutrition minérale et la fertilisation du Caféier sur sol schisteux en Côte-d'Ivoire. IV. Influence de la fertilisation minérale sur la composition du fruit. Café, Cacao, Thé, 47, 269-280

Verma TS, Tripathi BR (1987) Effect of Phosphorus levels on dry matter yield and micronutrients availability to Rice. Oryza, 24, 336-342

Viets $F G$ Jr, (1966) Zinc deficiency in the soil-plant system. In : Zinc metabolism. CC Thomas, Springfield, III, 123-146

Waring HD (1971) The nutritional status of pine plantation in Malaysia. Rep For Dept FAO, 49 
White MC, Decker AM, Chaney RL (1981) Metal complexation in xylem fluid. Plant Physiol, 67, 292-3to

Wilkinson $B$ (1957) Boron in the glasshouse Tomato plant. Nature, 180, 666

Wilkinson HF, Loneragan JF, Quirk JP (1968) The movement of Zinc to plant roots. Soil Sci Soc Am Proc, 32, 831-833

Will GM (1972) Copper deficiency in radiata pine planted on sand at Mangashai Forest. New Zeal J For Sci, 2, 217-221

Will GM (1978) Nutrient deficiencies in Pinus radiata in New-Zealand. NZJ For Sci, 8, 4-14
Will GM, Appleton EJ, Slow LJ, Stone EL. (1963) Boron deficiency. The cause of dieback in pines in the Nelson District. For Res Inst Rotorua Res, Leaflet, 1 , 1-2

Woods RV (1983) Trace element problems induced by heavy nitrogen fertilization of Pinus radiata in South Australia. Commun Inst For Fenn, 116, 178-182

Zyrin NG, Rerich WJ, Tikhomirov FA (1976) Forms of Zinc compounds in soils and its supply to plants. Agrokhimiya, 5, 124-131 